\title{
Pancreatic Tumor Metastasis of the Tongue
}

\section{Eda Kucukłulu, Uzer Kucukłulu', Ali Guner' ${ }^{1}$ H Bengu Cobanoglu², Sevdegul Mungan ${ }^{3}$,}

Abstract:

Distant metastasis to oral cavity is very rare and represents approximately $1 \%$ of all cases of neoplasm in oral cavity. Pancreatic cancers rarely metastasize to the oral cavity. Probably the highly aggressive nature of the pancreatic cancer does not give most of the patients a chance to live long enough to have an oral cavity metastasis. There is only a single case of pancreatic cancer with tongue metastasis reported in the literature. We presented a case of tongue metastasis of pancreatic cancer.

Key words: Pancreas, Tongue Neoplasms, Pancreatic Neoplasms, Mouth.

\section{Introduction}

Distant metastasis to oral cavity is very rare and represents approximately $1 \%$ of all cases of neoplasm in oral cavity [1]. In the oral soft tissues, the attached gingiva is the most common affected site, followed by the tongue and other oral soft tissues [2]. The prevalence of metastatic lesions in the tongue is reported to be $0.2 \%$ of 6881 cases of malignant diseases. The most common primary sites for oral metastases were the lung, kidney, liver and prostate for men; breast, female genital organs, kidney and colo-rectum for women [1]. Post-surgical distant metastasis is seen in $23-92 \%$ cases with 2 year survival rate being $10-20 \%[3,4]$.

Pancreatic cancers rarely metastasize to the oral cavity. Probably the highly aggressive nature of the pancreatic cancer does not give most of the patients a chance to live long enough to have an oral cavity metastasis. There is only a single case of pancreatic cancer with tongue metastasis reported in the literature [5]. The presented paper is the second reported case in the literature.

\section{Case Report}

72 years old male patient admitted to the hospital with the diagnosis of pancreas cancer with liver metastasis. Needle biopsy performed from the head of pancreas revealed ductal adenocarcinoma of pancreas. The patient was taken to a chemotherapy program. PET-CT evaluation after the completion of the chemotherapy protocol reported a $7 \mathrm{~cm}$ mass

\section{Corresponding Author: Prof. Uzer Kucuktulu}

Email: kucuktulu@yahoo.com

Received: July 30, 2013 | Accepted: September 26, 2013 | Published Online: October 30, 2013

This is an Open Access article distributed under the terms of the Creative Commons Attribution License (creativecommons.org/licenses/by/3.0)

Conflict of interest: None declared | Source of funding: Nil | DOl: http://dx.doi.org/10.17659/01.2013.0089 
located in head and corpus of the pancreas and solitary liver metastasis. It was classified as partial response to chemotherapy. Two months later, PETCT evaluation revealed a $2 \times 1 \mathrm{~mm}$ mass, hard in consistency in right anterior $1 / 3$ of the tongue [Fig. 1]. No evidence of tongue involvement was seen in PET-CT performed 2 months earlier. On contrast enhanced MRI, a $28 \times 25 \mathrm{~mm}$ sized mass with central necrosis components and with hypointense $\mathrm{Tl}$, hyperintense T2 signal characteristics were visible [Fig.2]. The biopsy of the lesion was reported as pancreatic adenocarcinoma infiltration [Fig.3].

When the age and performance status of the patient were considered, radiotherapy was decided to be the best treatment option. A total of $4320 \mathrm{cGy}$ in 180 cGy fractions with IG-IMRT technique in $\mathrm{Hi}-\mathrm{Art}$ Tomothreapy unit were given to mass localization of the tongue. The mass disappeared macroscopically without grade 3-4 side effects. After the completion of the radiotherapy the patient's conditions detoriated and expired 1 week after radiotherapy.

\section{Discussion}

Pancreatic cancer most frequently metastasize to lungs with only six cases of oral cavity metastasis reported [6,7]. The by-pass theory as Batson proposed may be effective in oral cavity metastasis of pancreatic cancers. According to Batson's theory, especially in cases of increased intrathoracic pressure, the venous flow is directed from the caval system to the valveless vertebral venous plexus which serves to by-pass filtering affect of the lung tissue and eases axial skeleton and head and neck metastasis [8].

It is extremely rare to see a tumor metastasis to the tongue. The prevalence of tumor metastasis to the tongue from other primary sites is $0.2 \%$. The possible metastatic routes to the tongue include arterial venous and lymphatic circulations. The most of the metastatic lesions prefer to lodge to the

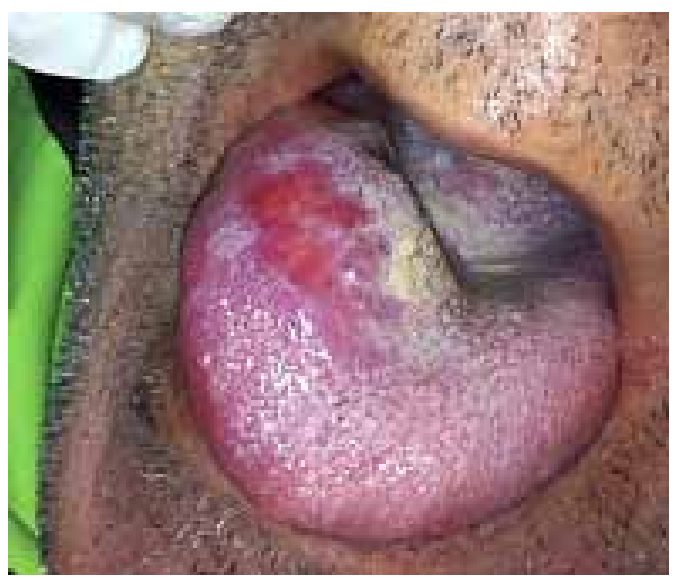

Fig. 1: Appearance of the metastatic lesion.

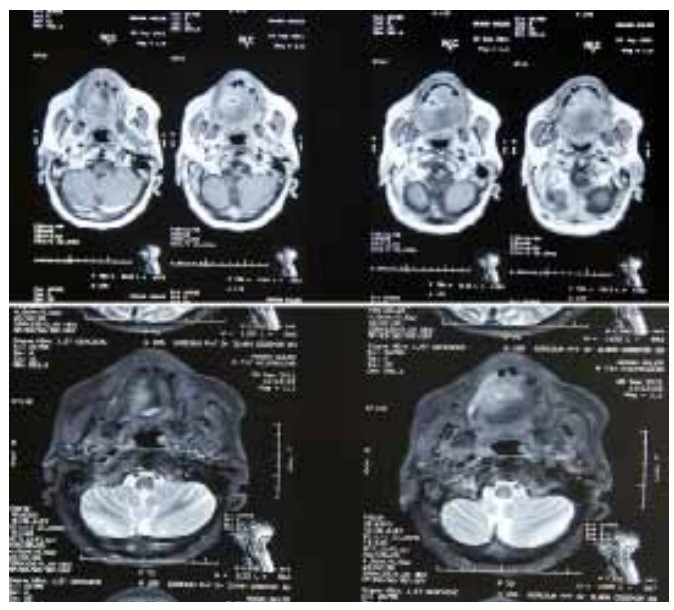

Fig.2: MRI appearance of the lesion.

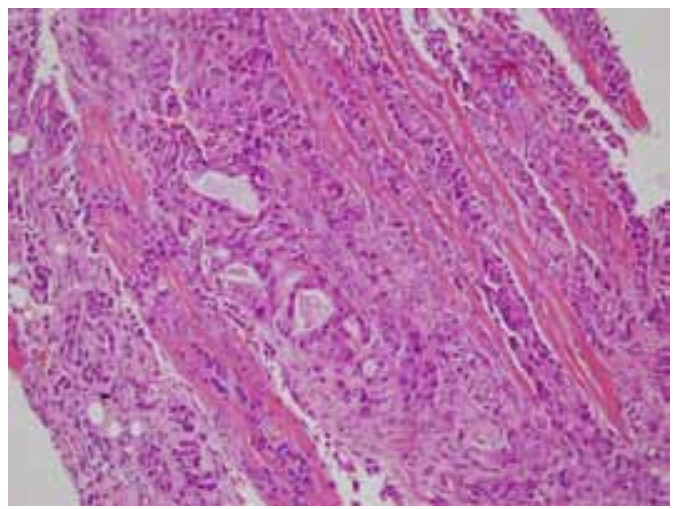

Fig.3: Adenocarcinoma infiltrating skeletal muscle of tongue (H-E x100). 
basal region of the tongue either because of its rich vascular supply of this area (via the dorsal lingual arteries) or relative immobility of this region of the tongue [9]. Despite the basal region of the tongue is the preferred site for the metastatic tumors, the primary tumors are most commonly seen in ventral and lateral sites of the tongue. The thinner mucosa with less surface keratin content of these sites potentially contribute to the higher incidence of primary tumors in ventral and lateral tongue. In the literature there are few reports of ventral tongue metastasis reported [10].

In our case, the metastasis was located to right ventral one third of the tongue. To our knowledge, it is the second reported case with ventral tongue metastasis. Although the tongue, gingivae and mandible share the same blood supply the preference of the tumor cells for certain sites for metastatic deposition suggest that there must be specific biological factors playing pivotal roles in determining this preference [11]. The inflammatory processes may be one of these factors attracting metastatic cells towards gingivae for colonization. There are evidences that chronic inflammation is related to various steps involved in tumourigenesis, including cellular transformation, promotion, survival, proliferation, invasion, angiogenesis and metastasis [12]. Although this theory is suitable to explain why primary cancers of tongue are seen in ventral and lateral tongue, it is far away to explain why metastatic depositions to tongue do not prefer same sites.

The mechanical, chemical and thermal alterations that the tongue is subjected may be a factor in inhibiting metastatic cell depositions. Further, several factors secreted from the skeletal muscle cells such as TNF- $\alpha$, TGF- $\beta$, lymphocyte infiltrating factor, interferon $\gamma$, lactic acid, proteolytic enzymes and adenosine receptor agonists are shown to have anticancer activity [13]. Despite all these, tongue is the second most frequent metastatic site of the oral cavity [2].

The cancer cells acquire capabilities to grow invasively and metastasize through multiple genetic and biochemical alterations of cell to cell and cell to matrix interactions. Tissue invasion and metastasis require, at different steps, contrary capabilities, for example, detachment from the primary tumor versus adhesion at the metastatic site, suggesting that rapid adaptations, genetic instability, and clonal selection play an important role [14]. The nonrandom pattern of metastasis in different types of cancer can be explained by "seed and soil" hypothesis describing the complex interplay between cancer cells and environmental factors [15].

The different primary tumors prefer specific sites in oral cavity to metastasize. The kidney and the lung cancers were the most common primary sites in tongue metastasis. It is not possible to determine the preferred site of metastasis in oral cavity for the pancreas cancer because of insufficient number of reports. There are only two cases of oral cavity metastasis of pancreatic cancer reported in the literature including this case.

Many primary cancers make distant metastasis to specific sites. Pancreatic and kidney cancers prefer to metastasize to the lungs [16]. The reasons for these preferences are not exactly known. But there are considerable evidences that the distant metastasis of various cancers is not a random event but it is a regulated site specific process [17].

The breast cancers metastasize most commonly to bones. It has been shown that osteolytic breast cancer cells over-express osteoclast-inducing factors such as PTHrP (parathyroid hormone-related protein], IL-8 and IL-1 1 [18]. These findings can explain the preference of breast cancer cells for metastasis to jaw bones in oral cavity. The factors that facilitate the oral cavity metastasis of pancreatic, lung and kidney cancers need to be investigated. As 
above mentioned evidences suggested there are many factors in the preference of the metastatic sites of the tumors. In locally advanced pancreatic cancer the life expectancy is quite short [4]. If the life expectancy in pancreatic cancer could be prolonged we probably would see more oral cavity metastasis. In pancreatic cancers, complicated chemocrine activities operate. These chemocrines effect both tumor survival and metastatic processes.

Although the chemocrine secretions of the tumors are well known we have little information about the chemocrine receptor expressions [19]. In the future new evidences could be reached about differences in metastatic biologies of lung and kidney cancer that metastasize to tongue more frequently versus pancreas cancers which makes metastatic tumor deposits to tongue rarely. If the factors effecting frequency of metastatic tumor deposits to oral cavity could be delineated, new targeted therapy methods for cancer with low survival rates such as pancreas cancers would be developed.

\section{References}

1. Hirshberg A, Leibovich P, Buchner A. Metastases to the oral mucosa: analysis of 157 cases. J Oral Pathol Med. 1993;22:385-390.

2. Hirshberg A, Shnaiderman-Shapiro A, Kaplan I, Berger R. Metastatic tumors to the oral cavityPathogenesis and analysis of 673 cases. Oral Oncol. 2008;44:743-752.

3. Mu DQ, Peng SY, Wang GF. Risk factors influencing recurrence following resection of pancreatic head cancer. World J Gastroenterol. 2004; 10:906-909.

4. Gastrointestinal Tumor Study Group: Treatment of locally unresectable carcinoma of the pancreas: comparison of combined modality therapy (chemotherapy plus radiotherapy) to chemotherapy alone. J Natl Cancer Ins. 1998;80:751.
5. Hassona $Y$, Hughes $C$, Prime SS. Letter to the editor. Metastatic tumours of the tongue. Oral Oncol. 2011 ; 47:308-311.

6. Cubilla AL, Fortner J, Fitzgerald PJ. Pancreas cancer-duct cell adenocarcinoma: survival in relation to site, size, stage and type of therapy. J Surg Oncol. 1978; 10:465.

7. Jemal A, Siegel R, Ward E, Murray T, Xu J, Thun MJ. Cancer statistics, 2007. Cancer J Clin. 2007;57:43-66.

8. Cummings J, Hacking N, Fairhust J, Ackery D, Jenkins JD. Distribution of bony metastases in prostatic carcinoma. $\mathrm{Br} J$ Urol.1990;66:411414.

9. Lang EE, Patil N, Walsh RM, Leader M, Walsh MA. A case of renal cell carcinoma metastatic to the nose and tongue. Ear Nose Throat J. 2003;82:382-383.

10. Torres-Carranza E, Garcia-Perla A, InfanteCossio P, Belmonte-Caro R, Laizaqa-Iriodo JM, Gutierrez-Perez JL. Airway obstruction due to metastatic renal cell carcinoma to the tongue. Oral Surg Oral Med Oral Pathol Oral Radiol Endod. 2006; 101:76-78.

11. Woodhouse EC, Chuaqui RF, Liotta LA. General mechanisms of metastasis. Cancer 1997;80:1529-1537.

12. Aggarwal BB, Shishodia S, Sandur SK, Pandey $M K$, Sethi G. Inflammation and cancer: how hot is the link? Biochem Pharmacol. 2006;72:16051621.

13. Bar-Yehuda S, Barer F, Volfsson L, Fishman P. Resistance of muscle to tumor metastases: a role for $\alpha 3$ adenosine receptor agonists. Neoplasia. 2001;3:125-131.

14. Hunter K. Host genetics influence tumour metastasis. Nat Rev Cancer. 2006;6:141-146.

15. Fidler IJ. The pathogenesis of cancer metastasis: the "seed and soil" hypothesis revisited. Nat Rev Cancer. 2003;3:453-458.

16. Motzer RJ, Bander NH, Nanus DM. Renal-cell carcinoma. N Engl J Med. 1996;335: 865-875. 
17. Pantel K, Brakenhoff RH. Dissecting the metastatic cascade. Nat Rev Cancer. 2004; 6:448-456.

18. Mundy GR. Metastasis to bone: causes, consequences and therapeutic opportunities. Nat Rev Cancer. 2002;2:584-593.

19. Balkwill F. Cancer and Chemokine network. Nature Rev Cancer. 2004;4:540-550. 